\title{
Saynètes de ménages. Le couple dans les romans de Jean-Philippe Toussaint
}

\author{
Isabelle BERNARD \\ Université de Jordanie \\ Département de Français \\ waelr@hotmail.fr
}

Recibido: 30/06/2012

Aceptado: 05/12/2012

\begin{abstract}
Résumé
Dans une perspective transversale et triaxiale, je vais présenter la vision toussaintienne du couple amoureux. Dans la première partie intitulée «Poulpes, amour et fantaisie», j'exposerai quelques photographies de couples. Dans «Dans la solitude des draps de coton », je traiterai plus particulièrement du personnage-narrateur, qui s'il n'est pas célibataire n'en demeure pas moins solitaire endurci : reclus ici ou là, salle de bain, cabinet de toilette ou cabine de téléphone, immergé dans la piscine d'un hôtel aussi profondément que dans ses pensées, il incarne l'individu hypermoderne aux prises avec son narcissisme et exprime les difficultés de vivre en couple comme en société. Enfin, une troisième partie, «Les alarmes d'Éros», plus exclusivement consacrée au cycle dédié à Marie, montrera la vision douce-amère des cœurs et des corps amoureux, saisie dans l'étroitesse des liens d'Éros avec Thanatos.
\end{abstract}

Mots clés : roman français contemporain, couple amoureux, sexualité, hypermodernité

\section{La pareja en las novelas de Jean-Philippe Toussaint}

\begin{abstract}
Resumen
En el presente artículo presentaremos la visión de Toussaint de la pareja de enamorados. Tras compartir en la primera parte del artículo, "Octopus, amor y fantasía", algunas fotografías de parejas de enamorados, pasaremos en la segunda parte, "En la soledad de las sábanas de algodón", a tratar más específicamente el narrador-personaje a quien el hecho no estar soltero no le impide ser un solitario empedernido, recluido en el baño, en el aseo o en una cabina telefónica, tan profundamente inmerso en la piscina de un hotel como en sus propios pensamientos. Es la encarnación del individuo ultramoderno que, enfrentado con su narcisismo, expresa las dificultades de vivir en la sociedad como pareja. Por último, una tercera parte, "Las alarmas de Eros", muestra la agridulce visión de corazones y cuerpos enamorados, captada en la estrecha relación de Eros y Thánatos.
\end{abstract}

Palabras clave: novela contemporánea francesa, la pareja en el amor, la sexualidad, la hipermodernidad

\section{The romantic couple in the novels of Jean-Philippe Toussaint}

\begin{abstract}
I will present the vision of Toussaint's loving couple. In the first part called "Octopus, Love and Dreams", I will present some photographs of couples. "In solitude in cotton sheets", I will discuss more specifically the character-narrator, who, despite the fact that he is not single, is none the less recalcitrant in his solitariness; a recluse in the bathroom, toilet or phone booth; immersed in hotel's
\end{abstract}


swimming pool as deep as in thought, he embodies the hypermodern individual struggling with his narcissism, and expressing difficulties of living with a partner. Finally, a third part named "The alarms of Eros" will show the bittersweet vision of hearts and body, interweaving Eros and Thanatos.

Keywords: Contemporary French novel, couple in love, sexuality, hypermodernity

\section{Referencia normalizada}

Bernard, I. (2013). "Saynètes de ménages. Le couple dans les romans de Jean-Philippe Toussaint". Thélème, Vol. 28, 27-44.

Sumario: 1. Poulpes, amour et fantaisie. 2. Dans la solitude des draps de coton. 3. Les alarmes d'Éros.

C'est grâce à un style épuré, d'aucuns diront minimaliste, et un ton narquois et désinvolte à souhait, que les huit romans de Jean-Philippe Toussaint, publiés par la maison Minuit depuis 1985, ont conquis universitaires et grand public ${ }^{1}$. Tendres et burlesques, tous font la part belle à une esquisse microsociologique des relations humaines. Depuis plus de vingt-cinq ans, dans une langue impeccable, à la fois soignée et précise, ironique et caustique, Toussaint étaie le portrait, filigrané de mélancolie et sans concession, d'un héros récurent, narrateur anonyme. De La Salle de bain jusqu'à La Vérité sur Marie en passant par Faire l'amour ou La Télévision, ce $J$ e, fidèle porte-parole de l'individu hypermoderne ${ }^{2}$, n'en finit pas de confier ses désirs et ses faiblesses, ses fantasmes et ses angoisses. Les femmes (Edmondsson, Pascale, Delon ou Marie) qui l'accompagnent, l'aiment, le quittent, lui permettent d'étoffer une interrogation existentielle sur les liens affectifs, fondateurs et puissants, mais précaires et aliénants.

Je me propose de présenter ces couples d'amoureux : d'abord dans une peinture de leur quotidien banal et anecdotique, ensuite dans un gros plan sur les difficiles relations d'altérité du Je, qui, s'il n'est pas célibataire n'en demeure pas moins solitaire endurci, et enfin dans une vision douce-amère (et teintée d'ironie) des cœurs et des corps, saisie dans l'étroitesse des liens d'Éros avec Thanatos.

\footnotetext{
${ }^{1}$ La Télévision a remporté le Prix Victor Rossel en 1997, Fuir le Prix Médicis en 2005 et La Vérité sur Marie le Prix Décembre en 2009.

${ }^{2}$ Selon Gilles Lipovetsky, l'hypermodernité définit le sujet contemporain dans une nouvelle étape du processus d'individualisation : chez le Je de Toussaint, elle se saisit notamment dans la mise en jeu de défenses narcissiques et dans une grande vulnérabilité à l'angoisse.
} 


\section{Poulpes, amour et fantaisie}

Toussaint inaugure son œuvre en esquissant un narrateur, pascalien en diable, à la sociabilité et à l'affectivité tourmentées : du fond de sa baignoire, le héros de $L a$ Salle de bain, moins impassible qu'inquiet et grave, raisonne à longueur de journée sur son être-là au cœur du Temps ou philosophe sur l'univers de signes qui l'entoure et qui peine parfois à faire sens. " À vingt-sept ans, bientôt vingt-neuf », le Parisien vivote, "exerçant en quelque manière la profession de chercheur " (Toussaint, 1985 : 15, 21). Son statut social mal déterminé allié à son allure adolescente, son émotivité, ses rêveries anxieuses et son absence de scrupules font de lui une « Sorte de Plume qui n'aurait pas entièrement renoncé à toute volonté de puissance ou d'homme sans qualités qui ne s'avouerait pas tout à fait comme tel» (Bessard-Banquy, 2003 : 20). À ses côtés vit Edmondsson qui subvient aux besoins de leur couple en travaillant dans une galerie d'art. Tous deux viennent d'aménager dans un nouvel appartement; aussi Toussaint creuse-t-il le banal de ce moment transitoire : apéritif avec les anciens locataires, aménagement, pendaison de crémaillère entre amis, choix de la couleur des peintures murales... Bien des scènes s'offrent comme de pures gourmandises narratives sans trop d'utilité diégétique. De sa plume ironique, l'écrivain en profite pour distiller quelques photographies de ce couple finalement peu ordinaire. Si Edmondsson et le narrateur composent un duo des plus disparates, leur connivence demeure partout éclatante car ils apprécient de s'amuser et rire ensemble. Hanté par une indicible anxiété liée à l'écoulement du temps, le $J e$ est cependant décrit tel un être renfrogné et angoissé, soumis à des sautes d'humeur, et conduit par sa recherche ataraxique à goûter le silence et la solitude de sa salle de bain. En contrepoint, quelques croquis dessinent sa compagne sensuelle et épanouie.

Une serviette de bain sur la tête, Edmondsson circulait toute nue dans la chambre, se déplaçant avec langueur, les seins en avant, avec de lents mouvements des bras qui, arrondis en l'air, décrivaient d'interminables spirales devant mes yeux (Toussaint, $1985: 20$ ).

La belle qui n'a pas sa langue dans sa poche use de son franc-parler de façon mordante; elle affronte les aléas du quotidien avec un aplomb que ne possède pas son compagnon. Elle incarne la femme nouvelle, une femme-sujet qui pimente aussi leurs relations conjugales :

[...] Un instant plus tard, Edmondsson apparaissait, le visage rayonnant. Elle voulait faire l'amour.

- Maintenant.

- Faire l'amour maintenant ? Je refermai mon livre posément, laissant un doigt entre deux feuil-

les pour me garder la page. Edmondsson riait, sautait à pieds joints. Elle déboutonna sa blouse (Toussaint, $1985: 17$ ).

C'est avec un style volontiers laconique que Toussaint croque les scènes d'amour, sous-entendant certainement que son cérébral narrateur privilégie l'esprit au corps. «Après avoir dénoué notre étreinte, nous restâmes un instant assis nus 
l'un en face de l'autre sur le tapis du vestibule » (Toussaint, $1985: 20$ ). Il réitèrera d'ailleurs cet effet dans La Télévision: "De retour dans la chambre, nous nous étions aimés (je n'en dirai pas plus, il est des moments où il faut savoir privilégier les faveurs de l'action aux agréments de la description » (Toussaint, 1997 : 257). Dans L'Appareil photo, le narrateur jouera de la même fausse pudeur (Toussaint, 1989 : 86). Pascale Polougaïevski n'a certes pas le dynamisme impertinent et jouissif d'Edmondsson, ni sa verve désarçonnante mais une langueur «adorable » (Toussaint, 1989 : 15) : elle n'est quasiment décrite que comme une "silhouette endormie » (Toussaint, 1989: 120), bâillant ou dormant « des plus pascalement » (Toussaint, 1989: 91). C'est néanmoins ainsi que la léthargique jeune femme attendrit son nouvel amant ! L'esquisse du couple est pour le coup complètement différente de celle présente dans le premier opus : le Je vient tout juste de faire la connaissance de cette mère de famille employée dans une auto-école et il multiplie les brefs instantanés sur un lien amoureux naissant. Attentions, caresses et étreintes répétées disent toutes les débuts d'une relation légère et rassurante. «Dans la cabine, pendant que nous descendions, je lui remis en place une mèche de cheveux. Elle me regardait. Je lui pris l'épaule, lui touchai la joue tendrement » (Toussaint, 1989 : 72). L'écrivain décrit un homme qui regarde une femme dont il est en train de tomber amoureux et travaille à conférer au ton de son narrateur ce goût indescriptible du printemps de l'amour ; ironique à l'envi, il force le trait afin de rendre son Je passablement béat.

Je me sentais tout dolent à côté d'elle (c'était peut-être l'amour déjà, qui sait, cet état grippal) ; Je proposai à Pascale de nous y arrêter pour boire un café, ou même un thé si elle voulait, j'étais prêt à tout. À tout (Toussaint, $1989: 28,88$ ).

Dans La Télévision, le romancier poursuit dans cette veine ironique son projet de décrire le quotidien sous ses aspects dérisoires et comiques ; il dessine les contours d'un autre $J e$ plus mûr, qui commence « tout doucement à avoir quarante ans » (Toussaint, 1997 : 11). Professeur à l'université, ce narrateur en congé sabbatique passe l'été à Berlin afin de rédiger un essai sur l'œuvre du peintre Le Titien sous l'angle des relations entre les arts et le pouvoir politique. Il a cependant gardé des précédents héros cette même allure indolente d'adolescent paresseux :

Depuis quelques mois, cependant, j'avais constaté une très légère dérive dans mon comportement. Je restais presque tous les après-midi à la maison, pas rasé et vêtu d'un vieux pull en laine des plus confortables, et je regardais la télévision pendant trois ou quatre heures d'affilée à moitié allongé dans le canapé [...] les pieds nus et la main sous les parties. Moi, quoi (Toussaint, 1997 : $10)$.

Émotionnellement, le Je semble plus apaisé, la présence de sa compagne enceinte, Delon (pour Madeleine), et de leur fils de 6 ans y est sans doute pour beaucoup.

L'engagement amoureux présente cette vertu, précieuse entre toutes, d'enrichir la vie subjective d'un horizon de sens [...] il permet d'échapper au désert du soi livré à lui seul. Lestant l'existence 
d'une dimension d'idéal et de sens, l'amour ouvre l'espoir d'une plus grande puissance d'exister par autodépassement de soi-même vers l'autre (Lipovetsky, 1997 : 50).

À l'image de toutes les amantes toussaintiennes, Delon, « lascive et princière » (Toussaint, 1997 : 266), est longuement admirée.

Elle était toute bronzée et déjà rieuse ma Delon, et elle s'avançait précautionneusement vers moi une main sur son chariot pour retenir les sacs, vêtue d'un pantalon noir et d'un tee-shirt blanc, enceinte, lumineuse, souriante, avec ses lunettes de soleil qui lui donnaient des allures de vedette de cinéma; Delon était nue, étendue dans le lit à côté de moi, le ventre immobile dans la pénombre, dont je devinais la peau lisse et tendue autour du nombril, et, la tête penchée sur le côté, elle me regardait dans les yeux avec un regard incroyablement droit et confiant. Je lui pris la main et la serrai tendrement (Toussaint, $1997: 250,257$ ).

C'est le pôle féminin qu'elle incarne qui joue le rôle de moteur dans le couple, d'où sans doute son surnom androgyne : Delon se place même en complète opposition face au Je. Active et épanouie, fière et pleine d'assurance, elle attend un second bébé alors que le narrateur est fréquemment décrit en plein délit de paresse, paralysé par la page blanche de son essai. S'il n'a au bout de l'été écrit que ces deux mots : «Quand Musset » (Toussaint, 1997 : 102), il aura largement commenté son apathie intellectuelle : «les chances que l'on a de mener un projet à bien sont inversement proportionnelles au temps que l'on a consacré à en parler au préalable » (Toussaint, 1997 : 54). Passif, il a effectivement préféré passer son temps dans un parc à lire distraitement, à vaguement méditer ou à « mater les corps nus de jeunes femmes allongées à proximité » (Toussaint, 1997: 61). Il est vrai qu'il était aussi tenu d'arroser les plantes des ses voisins alors qu'il essayait de se défaire de son addiction à la télévision! Malgré la tâche d'écriture qui lui incombe, il a volontiers opté pour de longues séances à la piscine où il a parfait sa brasse, " l'esprit idéalement vide» (Toussaint, 1997: 12). À lui de s'avouer régulièrement «tout occupé à ne rien faire », ce qu'il explicite ainsi : "Par ne rien faire, j'entends ne faire que l'essentiel, penser, lire, écouter de la musique, faire l'amour, me promener, aller à la piscine, aller cueillir des champignons » (Toussaint, 1997: 11-13)! Le narrateur profite de ces moments pour tirer toute une série d'anecdotes cocasses qui touchent ses vaines occupations dévoreuses de temps mais aussi des images de sa vie de famille : aussi laisse-t-il entendre les rires échangés avec son fils lors de leurs matches de football au milieu du salon ou poindre l'émotion née du contact de sa main sur le ventre arrondi de sa compagne (Toussaint, 1997 : 17, 102-103). Le Je sait toutefois se montrer sensuel et avoue grandiloquent : «J'aime beaucoup faire l'amour en effet [...] l'amour m'apporte un grand équilibre intérieur» (Toussaint, 1997 : 12-13). Ailleurs, il partage émotions et sensations sur le mode impassible, même si dans le flux des confidences, son humour se révèle parfois grivois :

Quel contraste, parfois, entre le simple taxi qu'on attend et les petites fantaisies sexuelles qui vous viennent à l'esprit. Les hanches et la chatte, sapristi ! Je me demandais bien pourquoi les hanches, d'ailleurs (la chatte, j'y voyais plus clair) (Toussaint, $1997: 144$ ). 
L'on notera que les prénoms des femmes (Edmondsson, Pascale ou Delon) qui, au fil des fictions, traversent l'existence des narrateurs prêtent à confusion, du fait de leurs connotations masculines : les femmes qui les portent sont pourtant loin d'avoir un physique androgyne. Dans ses derniers romans en date, Toussaint a également choisi un prénom évocateur puisque "Marie », anagramme du verbe aimer, est fort populaire et confère à l'héroïne un statut d'éternel féminin : elle figure ainsi une nouvelle Ève. D'une infinie modernité, à la fois "naïve et culottée » (Toussaint, $2002: 21$ ), hypersensible et mutine, la callipyge Marie s'avère le catalyseur du trio d'intrigues. Le narrateur se saisit de toutes les occasions pour la dessiner toujours plus vaporeuse et sensuelle.

Marie, en face de moi, adossée au mur, cambrée, les cuisses nues dans son tee-shirt blanc me regardait avec défi - il y avait du défi dans son regard, quelque chose de mutin, d'abandonné, de sexuel et de sauvage ; Marie, et son goût épuisant pour les fenêtres ouvertes, pour les tiroirs ouverts, pour les valises ouvertes, son goût pour le désordre, pour le bazar, pour le chaos, pour le bordel noir, les tourbillons, l'air mobile et les rafales (Toussaint, $2009: 59,15$ ).

C'est que lui aussi a des activités socioprofessionnelles réduites au minimum : qui est-il ? que fait-il dans la vie ?, le lecteur n'en sait presque rien. Aussi se targuet-il finalement de n'avoir qu'à dire La Vérité sur Marie ${ }^{3}$. «Je savais en toutes circonstances comment Marie réagissait, je connaissais Marie d'instinct, j'avais d'elle une connaissance infuse, un savoir inné, l'intelligence absolue : je savais la vérité sur Marie » (Toussaint, 2009 : 74). Le Je s'interroge sur son couple : il n'est plus tout à fait certain d'aimer Marie - rien n'est moins sûr dans son esprit en proie à moult tergiversations - mais il lui fait l'amour avec une passion attentive. Le corps de Marie, d'un port naturellement altier, est omniprésent et souvent dépeint abandonné, ce qui lui confère des allures de muse ancestrale égarée dans des lieux très modernes, hôtels ou musées. L'approche du couple a sporadiquement préservé l'aspect sentimental des premiers romans puisqu'elle empreinte d'identiques clichés et égraine les mêmes bribes amusantes d'un savoir-vivre amoureux (Toussaint, $2002: 14$ ). Si elle repose encore sur des métaphores et beaucoup de poésie, la prose a gagné en maturité et se montre plus sexuelle, violente et forte. Au fil des années, l'écriture s'est faite moins intellectuelle et plus sensuelle. Dans le cycle consacré à Marie, le narrateur, virtuose de la désillusion ludique, s'épanche moins sur l'éros que sur l'érosion de sa vie affective : il se laisse porter par un air du temps lancinant, teinté de renoncement et d'indolence. Sur fond de romantisme

\footnotetext{
${ }^{3}$ D'autant qu'il y en a deux dans le dernier opus : Marie, l'ex sublimée et inoubliable, et Marie, son double actuel et transitionnel, le narrateur narquois entretenant complaisamment la confusion. «Certes, je faisais clairement la distinction entre Marie et Marie - Marie n'était pas Marie -, mais j'eus immédiatement l'intuition que je ne parviendrais pas à me dédoubler moi-même » (Toussaint, 2009 : $38)$.
} 
urbain et récemment mondialisé ${ }^{4}$, le $J e$ se peint d'un trait à la fois trivial et poétique aux prises avec un vertige plus physique que métaphysique, celui des sens. Dans son "ars amandi », il narre avec un goût du détail cocasse ses élans sensuels et sexuels les plus marquants : les trames diégétiques laissent la parole à un homme qui exprime ses relations à la femme de sa vie qui a partagé son quotidien pendant six ans. Le couple vit séparé (c'est Faire l'amour qui raconte les interminables étapes de la rupture) mais demeure inséparable. Cette rupture jamais véritablement consommée, car toujours placée sous l'égide d'un désir renouvelé, constitue un point commun intertextuel. Une dose d'humour vigoureux, parfois féroce, travaille les jeux de l'amour et du désir qui tiraillent ce couple en désamour : le décalage comique dont l'incipit de La Vérité sur Marie donne à lui seul la teneur renforce les liens transtextuels. " Je me suis rendu compte que nous avions fait l'amour au même moment, Marie et moi, mais pas ensemble » (Toussaint, 2009 : 11). Blagues, situations absurdes, décalage burlesque, accumulation de clichés dévoilent la passion narrative du romancier quant à cette thématique. Amateur de croquis pris sur le vif, Toussaint aime à ridiculiser son narrateur en flagrant délit de coup de foudre. Dans Fuir, une étreinte dans les toilettes d'un train de nuit longuement préparée avec la douce Li Qi est brusquement interrompue par un appel téléphonique de Marie justement (Toussaint, 2005: 42) ; elle rappelle une scène d'amour torride -étape obligée et maintes fois répétée de la rupture - perturbée par l'arrivée d'un fax dans le luxueux hôtel tokyoïte de Faire l'amour (Toussaint, 2002: 34). De même, les retrouvailles des ex-amants dans le cadre romantique de l'île d'Elbe dans La Vérité sur Marie débutent par un épisode exempt de toute concupiscence : « Marie avait dû s'arrêter sur un promontoire, et j'étais sorti précipitamment de la voiture pour vomir (ah, quel séducteur, j'avais dû lui manquer) » (Toussaint, 2009 : 170). Derrière cet humour situationnel qui met au premier plan la formidable nonchalance du héros, il faut également déceler une singulière inhabileté à avoir vraiment prise sur le réel.

En fait, dans les fictions, c'est le pôle féminin du couple qui apparaît mature et fort ; il insuffle sa dynamique et sa sociabilité à son compagnon qui, bien souvent, n'en tire guère profit, ayant déjà bien du mal à composer avec lui-même. Toujours aux prises avec de complaisantes ratiocinations, le Je se trouve durablement empêtré dans une sourde solitude qui confine à l'incommunicabilité.

\footnotetext{
${ }^{4}$ Après des séjours en Italie et en Allemagne, le narrateur a franchi les océans et séjourne en Asie. Notons que la page d'accueil du site www.jptoussaint.com, créé par l'auteur avec la collaboration de Patrick Soquet, est une mappemonde sur laquelle sont inscrits les titres des romans en fonction des lieux diégétiques.
} 


\section{Dans la solitude des draps de coton}

Sous la plume de Toussaint, la vie conjugale induit des moments de complicité et de partage affectifs et érotiques mais également un immense vide philosophique. $\mathrm{Si}$ « le lien conjugal représente un segment primordial du lien social dans son ensemble » (Kaufmann, 1999: 125), il est très souvent délaissé par les héros ayant tous une nette propension au retrait et au solipsisme : les lieux les plus communs, clos de préférence (salles de bain, cabines de téléphone, toilettes, piscines...), exigus si possible, se constituent en "philosophoirs ", aptes à recueillir leur impérieux désir de se désolidariser du monde et d'autrui (voisins, collègues, amis ou connaissances) afin de soliloquer en paix dans l'immobilité la plus complète. « Seul dans un endroit clos, seul et suivant le cours de ses pensées dans le soulagement naissant, on passe progressivement de la difficulté de vivre au désespoir d'être » (Toussaint, 1989: 94). Certes, la sociabilité existe mais demeure insatisfaisante, et le critique agrée qui voit dans Toussaint un écrivain de l'abnégation :

Et c'est peut-être là l'art extrême de Toussaint que de pouvoir suggérer le charme triste des rencontres qui, loin de révéler une manière de multiplication de soi par l'autre, soulignent au contraire une sorte de division de tout et rendent plus évident encore l'isolement des êtres (Bessard-Banquy, $2003: 64)$.

Dans La Salle de bain, le nécessaire mais pesant repli sur soi du narrateur en perpétuelle méditation est porté à son paroxysme puisque dans une brève scène le $J e$ cédant à une pulsion d'agressivité plante une fléchette dans le milieu du front de sa compagne (Toussaint, $1985: 88$ ). Les relations de couple que le narrateur mène avec Edmondsson, par ailleurs décrites comme épanouissantes émotionnellement et sexuellement, s'avèrent terriblement en-deçà de ses attentes existentielles; elles se révèlent incapables de combler l'immense vacuité du monde qu'il ressent. Le héros ne cherche aucunement à se divertir, seul ou avec autrui, conscient que « tout le malheur des hommes vient d'une seule chose, qui est de ne savoir pas demeurer en repos, dans une chambre » (Pascal, 1941: 875). C'est le néant mort qu'il souhaite approcher tant il sait que « Notre nature est dans le mouvement; le repos entier est la mort » (Pascal, 1941 : 874). Malgré ses pointes de franche fantaisie, le roman par sa thématique autant que par sa composition serrée (trois parties intitulées « Paris, L'hypoténuse, Paris » découpées en paragraphes de longueur différente et tous numérotés) et sertie, si ce n'est de citations (Toussaint, $1985: 87$ ), de références aux préceptes pascaliens, retrace le quotidien d'un être passablement névrosé et rendu hypersensible par les leçons stoïciennes ${ }^{5}$.

\footnotetext{
${ }^{5}$ Dans L'Appareil photo, Toussaint reprendra sur le mode ironique cette inclination toute pascalienne (Toussaint, $1989: 31$ ).
} 
Il m'arrivait parfois de me réveiller en pleine nuit [...] et je posais la main sur le bras d'Edmondsson. Je lui demandais de me consoler. D'une voix douce, elle me demandait de quoi je voulais être consolé. Me consoler, disais-je. Mais de quoi, disait-elle. Me consoler, disais-je (to console, not to comfort) (Toussaint, $1985: 86-87$ ).

Interrogeant les paradoxes de l'infini au néant, de l'âme à la matière, de la vie à la mort ou encore de la vanité au sens, la bibliographie de Toussaint toute entière n'en finit pas de creuser les concepts et les Pensées de Pascal. Elle puise bien entendu dans des appréhensions plus modernes de ces paradoxes. L'approche barthésienne de la photographie comme «écrasement du temps : cela est mort et cela va mourir» (Barthes, 1980:150) confère ainsi une touche lancinante à une diégèse à l'origine des plus insignifiantes —et l'incipit en atteste : "C'est à peu près à la même époque de ma vie, vie calme où d'ordinaire rien n'advenait » (Toussaint, 1989: 7) - celle d'un Je qui souhaite s'inscrire dans une auto-école et a besoin de photos d'identité pour son dossier... Le « ça a été » (Barthes, 1980 : 140), cette interrogation sur le présent à jamais révolu qui n'est que la fuyante et vague réalité captée par la pellicule, fait basculer la fiction du côté de la métaphysique. Déjà vaguement obnubilé par la notion d'entéléchie, le narrateur trouve par hasard un appareil-photo (celui du titre, semble-t-il !) et, d'emblée, se l'approprie. Il fait quelques clichés de lui ${ }^{6}$ et, loin d'être apaisé des tourments du dehors, note à nouveau le lien entre l'absence de mouvement et l'inexistence. Et, si l'appréhension de la philosophie comme un refuge salutaire est récurrente chez Toussaint, c'est en particulier dans ce roman que se trouve l'illustration d'une thèse à laquelle fait allusion un calembour (Toussaint, 1989: 73) sur les marques d'essence et leur logos, d'une part, et sur la quiddité et le logos, d'autre part. L'essence de l'homme ne serait donc pas dans le discours mais dans la conscience, un flux de sensations et de pensées involontaires, à peine formulées, entre errances philosophiques et vaticinations. "La pensée, me semblait-il, est un flux auquel il est bon de foutre la paix pour qu'il puisse s'épanouir dans l'ignorance de son propre écoulement (Toussaint, 1989: 32). Les actes des protagonistes ne sont pas explicités : causes, raisons et volontés demeurent absentes. Et, de fait, la fiction rationaliste d'une délibération intérieure qui précèderait les actions et en déciderait est anéantie. La torpeur permanente du couple s'expliquerait par-là même, le sommeil étant non plus frère de la Mort, incertain Hypnos, mais plus sûrement une cruelle absence de volonté. Pascale plus que le narrateur, qui est toutefois croqué à plusieurs reprises comme « impassible et assoupi » (Toussaint, 1989 : 56), montrerait ainsi que la vie et les sensations sont essentielles alors que le logos et la pensée formulée accidentels. Si le narrateur constate plein de félicité que la jeune femme avec « une manière de langueur naturelle et foncière $[\ldots]$ oppos[e] en permanence à la vie une fatigue aussi sensation-

\footnotetext{
${ }^{6}$ Pascale appartient décidément à cette étendue immobile, car elle figure sur l'un des clichés, endormie.
} 
nelle » (Toussaint, 1989:84), c'est qu'il l'envie, son aspiration à se soustraire à l'action délétère du temps se faisant impérieuse. À lui d'adopter ce vade mecum: « dans le combat entre toi et la réalité, sois décourageant» (Toussaint, 1989 : 50).

La Télévision est un roman plus étoffé que les précédents mais qui déploie le même ton à la fois désinvolte et provocateur. La solitude du Je y semble plus légère à envisager et doit être profitable à un travail de recherche. Le plaisir naît pourtant rarement de ces moments peu productifs égrenés tout l'été par le héros. L'inquiétude identitaire de cet « individu incertain » ${ }^{7}$ sourd derrière son incapacité à se mettre au travail. Son essai restera « une éventualité délicieuse et lointaine, un peu vague et abstraite, rassurante, que les circonstances, malheureusement, [1'] empêchaient de mener à bien pour l'instant (Toussaint, 1997 : 193). Velléitaire, le $J e$, préfèrera de loin « rêvasser aristotéliciennement» (Toussaint, 1997: 89). Et on le verra accaparé par une somme d'agacements ridicules ou d'exigences improbables qui le conduiront invariablement à conclure que « dans la perspective même d'écrire, ne pas écrire est au moins aussi important qu'écrire » (Toussaint, 1997 : 90). S'il désire cesser de regarder la télévision sans pour autant y parvenir, c'est aussi parce qu'elle incarne un divertissement au sens pascalien du terme avec lequel il a tressé un lien indéfectible et passionnel. Cette mauvaise compagne des heures perdues accentue son inactivité qui l'inhibe déjà et peu à peu le déprimera complètement (Toussaint, 1997 : 231). Le protagoniste de La Télévision semble profondément perturbé par des angoisses métaphysiques et lui aussi n'a de remède pour se soustraire à la pesée du monde que le refuge de sa baignoire ou du bassin d'une piscine. Certes, la clausule montre sa famille enfin réunie et le décrit détendu et tout à fait heureux de la complicité retrouvée tant avec son fils qu'avec Delon. Il lui faut pourtant attendre que la maisonnée soit endormie pour ressentir cette quiétude depuis longtemps espérée. La plénitude, sorte d'ataraxie enfin atteinte, se savoure, comme il se doit, dans un isolement immobile et silencieux (Toussaint, 1997 : 270).

Le $J e$ a somme toute un rapport très ambigu et très ambivalent à la présence de l'autre féminin: il s'agit d'un autre paradoxe existentiel. Dans Faire l'Amour, lorsque Marie s'absente, elle n'en cesse pas moins d'envahir l'espace alentour et hante la conscience de son amoureux. Aussi, dans la scène finale, le narrateur pénètre-t-il dans l'obscurité le musée qui accueille l'exposition des robes et des portraits de Marie pour se rapprocher d'elle après une séparation de plusieurs jours. L'aura de la styliste confère à cette visite impromptue son caractère fantomatique et obsédant. «Marie était là. Ce ne fut pas à proprement parler une hallucination, car la scène eut lieu en dehors de toute représentation visuelle, mais dans un registre purement mental » (Toussaint, 2002 : 173). Le narrateur a, en effet, ouvert le flacon d'acide et le tient devant lui, menaçant de ses vapeurs malsaines : il se dégage dans l'atmosphère symbolisant l'être de Marie et entourant ses créations «altières,

\footnotetext{
${ }^{7}$ L'expression est du sociologue Alain Ehrenberg (1996) L'individu incertain. Paris, CalmannLévy.
} 
décolletées, séductrice et colorées, amarante, incarnadines » (Toussaint, 2002: 2223) des substances destructives. N'adressant ordinairement à sa compagne que constats mièvres ou insultes, il garde pour lui seul aveux et déclarations d'amour. Derrière la valeur accordée à l'instabilité et l'insatiabilité du désir, la labilité des liens interpersonnels s'exprime encore une fois telle une interrogation angoissée et obsédante. De fait, la hantise du Je concerne moins sa sexualité que sa solitude ; elle rend plus tangible encore le sentiment croissant d'insuffisance et de dépréciation de soi qui repousse la survenue de l'épanouissement. Ainsi n'est-il pas anodin que dans Faire l'amour, le narrateur bataillant avec ses pulsions sadomasochistes porte constamment dans la poche un petit flacon d'acide chlorhydrique. « Marie se demandait, avec une inquiétude peut-être justifiée, si ce n'était pas dans mes yeux à moi [...] que cet acide finirait. Ou dans sa gueule à elle» (Toussaint, 2002:11). Par contrecoup, la sérénité du couple reste en devenir, contredisant le désir des corps en quête de jouissance immédiate. Replacée dans le cadre narratif des intrigues, la séparation d'avec Marie se lit telle une étape dans la narration d'une histoire d'amour en déconstruction autant qu'en reconstruction.

Le couple a changé. Autrefois institution dans laquelle on entrait pour la vie sans trop se poser de questions, il est devenu un système mouvant d'ajustements permanents de la vie à deux et requiert désormais un véritable travail et une compétence de la part de ceux qui tentent l'expérience (Kaufmann, $1999: 125$ ).

Logiquement, l'échec sentimental devient une véritable aventure à péripéties, qui atteste d'une vaste désorientation émotionnelle : «Rompre, je commençais à m'en rendre compte, c'était plus un état qu'une action, un deuil plus qu'une agonie » (Toussaint, 2002: 129). Dans cette description de sa rupture, le narrateur observe minutieusement l'action délétère de ses sentiments, de ses élans et de ses envies, en même temps qu'il scrute ceux de sa compagne avec une acuité et une lucidité surprenantes.

J'avais le sentiment qu'elle se servait de mon corps pour se masturber contre moi [...] et c'était sans doute exactement le même sentiment qu'elle devait éprouver envers moi, car, moi aussi, depuis que notre bras-le-corps était devenu cette lutte de deux jouissances parallèles non plus convergentes mais opposées, antagonistes, comme si nous nous disputions le plaisir au lieu de le partager, j'avais fini par me concentrer comme elle sur une recherche de plaisir purement onaniste (Toussaint, 2002:33).

Soulignant la solitude inhérente à l'humain, les intrigues se fondent sur le délitement d'un couple d'amoureux fragiles et désorientés affectivement. Si elles contiennent tous les bribes d'un discours sentimental et les fragments d'une esthétique romantique animés par un Je nonchalant, parfois très amusant, elles sont néanmoins traversées par une tension dramatique pesante car, épris de paradoxes et d'apories philosophiques, les narrateurs y sont fréquemment submergés par une vague de nostalgie et de tristesse, suite à leurs réflexions continues sur le passage du temps, la quiddité et la mort. L'approche des liens affectifs s'avère des plus tourmentée : Toussaint a le don de composer avec le doux-amer. 


\section{Les alarmes d'Éros}

Les fictions de Toussaint, intimistes, travaillent les homologies entre espaces et personnages; elles disent l'appréhension toujours subjective des lieux où se fondent les liens entre l'amour et la mort. Chaque diégèse qui compte bien des dialogues décharnés - et, par exemple, le Je de La Salle de bain n'aime rien tant que partager de « longs silences ensemble» avec Edmondsson (Toussaint, $1985: 67$ )— offre un cadre particulier aux moments introspectifs ; elle possède sa thématique propre et indépendante, ses couleurs et ses paysages : sa géographie sentimentale singulière.

En raison du succès international du roman, la critique a longuement glosé le cadre neutre et hygiéniste dévolu à l'intrigue de La Salle de bain comme elle a disséqué les propos et pensées de l'Archimède des temps postmodernes qui y gît. Il est vrai que, malgré une échappée milanaise, le cadre spatial évolue peu. L'intermède italien, intitulé « L'Hypoténuse $»^{8}$, présente une sorte de léger dépaysement, à peine une distraction: la définition mathématique de ce mot-clef permet de comprendre que c'est par l'hypoténuse que l'on s'éloigne du vif du sujet mais aussi par elle que toujours on y revient ! Ce serait donc un autre moyen de toujours mieux « fatiguer la réalité ». À ceci près qu'il demeure vain : l'enfermement, la sortie milanaise puis le retour au refuge-baignoire désignent par excellence un mouvement illusoire, un retour au même qui marque l'absence de toute perspective face au destin. Propice à la méditation, la salle de bain n'en reste pas moins un lieu désincarné où le couple se rencontre cependant mais où surtout le narrateur dans ses périodes de crise antisociale s'adonne seul à ses rêveries pleines d'anxiété. Par définition, cet espace réduit n'est pas destiné à la socialisation. La mère du narrateur y viendra toutefois ainsi qu'un ami de ses parents (Toussaint, 1985 : 13-14). Ce cadre original incarnerait ce moment de délitement de la sociabilité que traverse le $J e$, même s'il a été réaménagé : certes, le miroir et les fissures des murs blanchâtres accentuent les possibilités de fixer son esprit sur l'infini mais le narrateur a tenu à y placer une partie de sa bibliothèque (Toussaint, $1985: 12$ ).

La philosophie n'est pas la seule discipline à tenir une place de choix dans cette singulière diégèse : la peinture s'y trouve omniprésente, et la connaissance des arts est perçue telle une activité intellectuelle importante, à la source de réflexions philosophiques. Parmi les nombreux artistes cités, l'on relève le néerlandais Mondrian aux origines de l'abstraction géométrique. La symétrie colorée de ses toiles séduit d'abord par sa solennité : en quête d'une pureté absolue, son art ne pouvait que fasciner le narrateur, scrupuleux observateur, attentif aux harmonies de formes et de tons, au point de l'obséder.

Je songeais à la dame blanche, le dessert, boule de glace à la vanille sur laquelle on épanche une nappe de chocolat brûlant. Depuis quelques semaines, j'y réfléchissais. D'un point de vue scienti-

\footnotetext{
${ }^{8}$ Le théorème de Pythagore sert d'exergue au roman (Toussaint, $1985: 7$ ).
} 
fique (je ne suis pas gourmand), je voyais dans ce mélange un aperçu de la perfection. Un Mondrian. Le chocolat onctueux sur la vanille glacée, le chaud et le froid, la consistance et la fluidité. Déséquilibre et rigueur, exactitude (Toussaint, $1985: 14-15$ ).

Le moindre geste, celui de se raser (Toussaint, $1985: 25$ ) par exemple, prend dès lors des proportions insoupçonnables, puisque c'est encore la patte de Mondrian qui soutend ce mouvement. La facture nette et lisse des toiles du maître de l'abstraction, déclinant en priorité à côté du blanc et du noir trois des couleurs primaires (rouge, bleu et jaune) organisés en savants agencements orthogonaux, a bel et bien de quoi ravir le narrateur dans son obstination funeste à vouloir saisir l'immobilité de tout mouvement, à vouloir, plus prosaïquement dit, maîtriser l'espace et le temps qui sont les coordonnées précaires de l'existence humaine.

Ce qui me plaît dans la peinture de Mondrian, c'est son immobilité. Aucun peintre n'a voisiné d'aussi près l'immobilité. L'immobilité n'est pas l'absence de mouvement, mais l'absence de toute perspective de mouvement, elle est mort. La peinture, en général, n'est jamais immobile [...] Chez Mondrian, l'immobilité est immobile. Peut-être est-ce pour cela qu'Edmondsson trouve que Mondrian est chiant. Moi, il me rassure (Toussaint, $1985: 84$ ).

Apte à être lue telle une fable philosophique, La Salle de bain, explicite également en son sein le paradoxe de l'immobilité dans le mouvement en introduisant une relation directe entre les fléchettes du héros (avec lesquelles il mime notamment la mort d'Edmondsson, la visant sciemment) et celles qui ont servi à la démonstration restée célèbre d'un disciple de Parménide, le philosophe grec Zénon d'Élée, qui concluait à l'immobilité d'une flèche lancée dans l'air en raison de l'inexistence même d'un état de mouvement. Mais, bien entendu, cette fléchette qui blesse la femme aimée symbolise l'immanquable prégnance du mortifère dans la relation amoureuse.

L'Appareil-photo reprend selon une approche légèrement différente les réflexions de $\mathrm{La}$ Salle de bain, puisque le narrateur apprécie plus que tout être « idéalement nulle part, si ce n'est immobile dans [son] esprit» (Toussaint, 1989: 102). Ses mini-aventures (qui consistent notamment à faire des photos d'identité pour son dossier d'auto-école et passer un week-end en amoureux en Grande-Bretagne...) vont rapidement lui donner l'occasion de traverser différents non-lieux, à commencer par des lieux de transit «propices à une réflexion sur ces paradoxes de l'immobilité mouvante et du mouvement immobile» (Schoots, 1997: 152) : gares et aéroport, routes, grâce à plusieurs moyens de transport (voiture, avion, bateau et train) pour ses déplacements Outre-Manche et en banlieue parisienne (une équipée le mène avec Pascale et son père à Créteil à la recherche d'une bouteille de gaz). Le week-end à Londres n'est pas des plus pittoresques. Le décor est sobrement dépeint : il est fait mention d'un froid vif et de pluie. Les activités touristiques sont réduites au minimum, l'attente d'une table dans un restaurant indien prenant le pas sur toutes les autres découvertes des amoureux. Les quelques mots et expressions anglaises qui émaillent le récit lui confère finalement son seul exotisme. En fait, cet épisode n'est que prétexte à redire l'immense tendresse que le $J e$ voue à Pascale. Les trajets sont plus que les lieux porteurs de sens. Quant aux objets, ils accentuent 
la thématique duelle de l'immobilité et la tendance à l'auto-contemplation du narrateur. À l'instar du miroir, souvent cité —et l'on sait que toute expérience du miroir est lié au sentiment de mort-, l'appareil-photo qu'il a trouvé sur le bateau le menant de Newhaven à Dieppe, sert au narrateur à mesurer le tragique passage du temps sur son corps et sur son visage. De cette conscience de la finitude, provient sans nul doute son désir d'échapper à la réalité définie comme l'écoulement du temps amenant à l'inacceptable dégradation de toute vie. Et, sur les photos d'identité qu'enfin il parvient à faire, il observe une "sorte de lassitude dans la manière d'être là » (Toussaint, 1989: 96); elle rappelle cet épisode de La Salle de bain qui voyait le $J e$ en Hamlet moderne s'égarer dans l'observation de son crâne sur une radiographie médicale (Toussaint, 1985 : 99). Lieux et objets mais aussi personnages de l'intrigue, tous travaillent ainsi une thématique semblable qui rapproche l'amour de la mort. Aussi, le prénom de la somnolente fiancée, Pascale, est-il un renvoi en forme de clin d'œil au philosophe auvergnat qui se trouve également au cœur du premier opus, même si c'est bien le narrateur obsédé par l'inévitable réalité qui porte en lui la sensibilité pascalienne. À ses côtés, la belle endormie est délivrée de tout projet et échappe à toute pensée existentielle sur l'irrémédiable écoulement du temps en s'absentant du monde par un sommeil protecteur. Ainsi indolente, elle se réalise dans l'exclusion de tout dynamisme volontaire et mime l'absence de mouvement, cette immobilité que son compagnon n'a de cesse de vouloir saisir. La clausule (Toussaint, 1989 : 126-127) dépeint le narrateur dans une position de recueillement avec toujours le même et inextricable nœud conceptuel à l'esprit : au fond d'une cabine téléphonique sise sur le bord d'une route de la campagne orléanaise, dans laquelle il a vainement attendu toute la nuit un appel de Pascale (qui s'est probablement endormie) dans un de ces moments de désespoir d'être absolu, le voilà qui raisonne et métaphorise son idée fixe et toute entière fantasmatique.

Il faut bien avouer que La Télévision ne contient pas de pauses aussi tragiquement tristes et tourmentées. L'intrigue se déroule en Allemagne et le roman offre ainsi de surprenantes photographies de la capitale dans la touffeur exceptionnelle d'un été car Berlin et ses «immenses concentrations de blocs d'immeubles grisâtres » (Toussaint, 1997: 214-221). La vision globale d'une capitale en travaux interpelle le narrateur qui n'aura pas le loisir de développer de façon anthropologique ses constats. Redescendu sur la terre ferme, il pose en revanche un vrai regard d'ethnographe en vacances sur la population estivale du verdoyant parc Halensee, halluciné de lumière et dévolu à la détente et aux loisirs, qui sert d'écrin à ses longues après-midi de farniente. Sur la pelouse, il côtoie quelque quatre cents promeneurs qu'il croque de son implacable coup de crayon narquois, le narrateur toussaintien étant comme à l'accoutumée impitoyable avec ses contemporains. Il les dessine "pour la plupart nus », tel un conglomérat de chair humaine, de quelques rares morceaux de tissus, de poils de chiens, de roues et de roulettes et autres cadres de bicyclette (Toussaint, 1997 : 60-61). Sous le soleil, les corps abandonnés attisent bien des tentations de ce promeneur solitaire et, d'un coup, réveillent son instinct de séducteur autant qu'ils suscitent de frustrations. Poussé par l'irrésistible chaleur qui 
l'accable, étendu et détendu sur l'herbe rase, le chercheur en panne d'inspiration ira donc jusqu'à contourner les "convenances vestimentaires urbaines" (Toussaint, 1997 : 64) et, toute sa pudeur surmontée, finira complètement nu par traverser le parc en direction du lac où une salutaire baignade, rafraichissante pour le corps et l'esprit, mettra fin à la scène (Toussaint, 1997 : 67-76). L'humeur du Je, assumant sa maturité nouvelle tout autant que sa nudité, s'est faite plus gaie: si ses propos - les difficultés d'écrire inlassablement répétées - demeurent anxiogènes, ils sont compensés par beaucoup d'autodérision. C'est dans l'obscurité du salon où trône le téléviseur qu'il faut saisir le lieu clef de l'intrigue car, parallèlement à la rédaction de son essai, le narrateur a fait vœu d'éteindre définitivement sa télévision. Ce souhait le conduit à se dévoiler plus avant car c'est dans ce lien au petit écran, fidèle compagnon de son été allemand, que le narrateur se portraitise le plus justement et donne de l'épaisseur à son regard sur l'ère actuelle, l'absence de Delon ne suscitant pas une telle tristesse. L'approche de l'accaparant téléviseur, tel un abîme, imprime au roman léger, burlesque et plein d'allant une imperceptible touche mortifère. Le $J e$ essaie de cerner au mieux son addiction, son « ivresse mauvaise » :

Sans pouvoir réagir, j'avais conscience que j'étais en train de m'avilir en continuant à rester ainsi devant l'écran, la télécommande à la main que je ne pouvais lâcher, à changer de chaîne machinalement, frénétiquement, dans une recherche de plaisirs immédiats et mauvais, entraîné dans cet élan vain, cette spirale insatiable, à la recherche de plus de bassesse encore, davantage de tristesse (Toussaint, 1997: 22).

Comme les miroirs ou les photographies des premiers romans, la télévision accentue le penchant du narrateur à l'auto-contemplation; dans les œuvres suivantes, l'espace romanesque tout entier sera devenu un reflet de son âme mortifère.

L'intrigue de Faire l'amour se situe en effet au cœur de la capitale japonaise, dépeinte dans des compositions diurne et d'aube, de soleil bleuté se levant dans une pureté glaciale ou de jour tombant lacéré de gris sous une pluie, froide et incessante. C'est avec un regard d'insomniaque que le $J e$, éreinté par le décalage horaire, y observe Marie. L'écriture qui soigne ce décor aux nombreux reflets —entre miroirs et flaques - possède ce qu'il faut de tension diffuse, aidée en cela de quelques légères secousses telluriques, peut-être annonciatrices d'un tremblement de terre plus destructeur. Elle file une métaphore liquide allant des pleurs à la pluie en passant par l'acide chlorhydrique. Le liquide corrosif mène à un parallèle explicite et inattendu qui traduit la douleur du $J e$, l'absence de fusion charnelle et la négation de l'autre, façon d'autrement dire l'inséparable duo composé d'Éros et de Thanatos. «Et à mesure que l'étreinte durait que le plaisir sexuel montait en nous comme de l'acide, je sentais croître la terrible violence sous-jacente de cette étreinte » (Toussaint, 2002 : 34). La sexualité exprime les soubassements de la personnalité et la

\footnotetext{
${ }^{9}$ Une scène a lieu dans une salle de bain qui rappelle par son approche les thèmes abordés dans le même cadre dans le roman La Salle de bain (Toussaint, 1997 : 256).
} 
part inhérente à la nature humaine où chacun, finalement, ne vit que pour soi. L'auteur conduit son lecteur au-delà de la rupture amoureuse et l'amène à réfléchir sur le sens du plaisir et de la passion, sur les principes qui déterminent le désir qui meurt avec le temps ou qui se dissocie du sentiment amoureux, laissant l'être face à son néant. De fait, la tension entre les amants est palpable tout autant que l'inquiétude identitaire: saisi dans des postures qui trahissent son vœu d'anéantissement, le narrateur livre un combat contre des pulsions de mort : « Moi nu dans les ténèbres de la salle de bain qui jetais de toutes mes forces l'acide chlorhydrique à la gueule du miroir pour ne pas voir mon regard » (Toussaint, 2002:42). L'ascendant que Marie possède sur lui comporte un degré certain de dangerosité puisque, sans être paranoïaque, il se décrit dans l'œil d'un terrible cyclone homonyme (Toussaint, 2002: 167) ! Filant plus avant la métaphore climatique, le narrateur en vient tout bonnement à souhaiter la fin du monde : devenant totalement étranger à lui-même dans ces instants, il envisage sa propre destruction, et avec elle celle de toute la planète, comme unique remède adéquat et à la hauteur de ses tourments (Toussaint, $2002: 69$ ).

Symétriquement, Faire l'amour se place sous le signe d'un Japon tiraillé entre ses extrêmes, à la fois hypermoderne —avec ses hôtels panoramiques impersonnels et silencieux, comme insonorisés et ses musées d'art contemporain futuristes- et fort traditionnel avec les minuscules restaurants à soupes du quartier Shinjuku où se déguste une cuisine du cru et ses paisibles sanctuaires millénaires, tel celui de Kyoto visité par le narrateur (Toussaint, 2002 : 157). L'étape tokyoïte s'étiole sur cette constatation désolée :

Nous nous aimions, mais nous ne nous supportions plus. Il y avait ceci, maintenant, dans notre amour, que même si nous continuions à nous faire dans l'ensemble plus de bien que de mal, le peu de mal que nous nous faisions nous était devenu insupportable (Toussaint, $2002: 82$ ).

Le roman Fuir peint, quant à lui, plusieurs tableaux vivaces d'une Chine indomptable et exotique, explorant le thème des pulsions du désir et du manque à l'aide d'une palette variée de tons chatoyants et de reflets délavés. Certes, dans le début du roman, le narrateur se consacre peu à ses relations distendues avec Marie tant il est pris dans la dynamique de son infatigable hôte, qui le mène tambour battant à travers le pays. Cependant, l'équipée aussi incongrue que drôle mixe un étonnant fatras de lumières, de bruits et d'odeurs envahissantes, d'impressions, de sensations où se mêlent et s'emmêlent, sur un mode hallucinant et discontinu, incompréhension, peur panique et érotisme. Et c'est la douce Li Qi qui symbolise l'étonnant rapport, délicieusement frustrant, du narrateur à la Chine (Toussaint, 2005 : 37-38). À partir de la troisième partie se développe un récit de deuil lorsque survient la disparition du père de Marie. Au défilé rapide et haletant d'images et d'émotions, succède alors une pause descriptive dans laquelle la peinture figée des paysages naturels de l'île d'Elbe s'accorde à la fébrilité du narrateur et à l'émotion contenue de sa compagne. Le paysage de bords de mer écorchés aux couleurs lumineuses, avec les pierres des maisons saturées de lumière se mariant aux tons éclatants des lauriers-roses et des bougainvilliers s'offre tel un écrin au chagrin des 
personnages. La clausule porte à son paroxysme la géographie sentimentale : « Marie pleurait dans mes bras, dans mes baisers, elle pleurait dans la mer » (Toussaint, 2005 : 186). Le potentiel comique contenu dans le décalage des cultures lors de l'épisode chinois s'oppose totalement au contenu dramatique de l'épisode italien dans lequel les émotions restent néanmoins toutes aussi impossibles à partager. Dans une odeur de cierges brûlés se joue un drame intime qui fige dans l'instant Marie et son compagnon incapables de suppléer une langue impuissante : «Elle était seule dans sa douleur, et j'étais seul dans la mienne » (Toussaint, $2005: 169$ ). Entièrement soumis à cette esthétique du décalé, le roman est parcouru d'une tension ironique, car dans les propos glacés ou dans les descriptions dramatiques persiste la possibilité d'un second degré qui annulerait toute émotion négative. C'est également le cas de Faire l'amour qui contient de ces déplorations indolentes annulant le potentiel érotique du titre. "Qu'avais-je à faire ces jours-ci à Tokyo ? Rien. Rompre » (Toussaint, $2002: 129)$.

La diégèse de La Vérité sur Marie mixte à son tour thème, couleurs et décors pour exprimer la tension amoureuse ; elle repose sur trois grands espaces, travaillés tels des paysages nocturnes, noirs et bleutés, tous éclairés artificiellement. C'est dans la première partie du roman que la tension entre Éros et Thanatos est la plus édifiante, à ceci près qu'elle ne concerne pas le couple formé par Marie et le narrateur, mais celui formé par Marie et son nouveau compagnon. Dans l'appartement parisien de sa maîtresse, rue de la Vrillière, ce dernier succombe à une crise cardiaque. Si quelques instants auparavant, les éclairs d'un violent orage d'été illuminaient brièvement les corps amoureux, impatients de s'éteindre, ils étaient à lire comme les signes avant-coureurs d'un drame d'une extrême violence et soudaineté : le décès d'un homme. L'incipit chargé de vibrations négatives n'était donc pas le seul fait de la jalousie du narrateur ni les conséquences d'une touffeur nocturne malsaine «moite, irrespirable et délétère » (Toussaint, $2009: 22$ ). L'ensemble de la scène rend compte d'une inquiétude diffuse et oppressante fort inattendue dans le vif d'une relation sexuelle dépeinte par le menu (Toussaint, 2009 : 18-22) mais qui trouve son point d'acmé avec la description des symptômes d'un malaise cardiaque. Évoquant la rencontre de Marie avec ce nouveau compagnon, le Je narre dans le chapitre suivant une scène épique. Le flash-back renvoie à l'intermède japonais de Faire l'Amour : sur le tarmac de l'aéroport de Narita lors du retour vers Paris, Marie et son amant convoient un cheval. Sur près de vingt pages, Toussaint le laisse galoper, aveuglé par les projecteurs de la police, ce fougueux cheval nommé Zahir alors qu'il vient de s'échapper de son van. Il fait de cet épisode scénarisé comme pour une prise de vue cinématographique en long travelling un emblème de la relation amoureuse ténébreuse du $J e$ avec Marie. S'y mêlent la pluie, la peur et la panique : Marie a égaré son passeport et le pur-sang a disparu! L'absence du narrateur sur place est supplée par ses talents de conteurs cocasse. Flamboyante, cette seconde partie de la diégèse garde néanmoins les traces d'une mélancolie tokyoïte plus avant décrite et associée à la rupture. Cependant, c'est la fin du roman qui constitue le moment apocalyptique redouté : un feu de forêt, qui place définitivement la trilogie sous l'égide des Éléments, dévaste au petit matin les flancs arides 
de l'île d'Elbe, précisément la colline sur laquelle sont sises la villa et les écuries de Marie. Jusque là, le lieu paradisiaque, même s'il était encore pour Marie placé sous le signe de la mémoire du deuil paternel, composait le cadre enchanteur et ensoleillé d'un délicieux jeu de la séduction toujours recommencé entre les ex-amants, tout en frôlements et élans de désir refoulés, qui aiguisaient leurs sens. Mais, alors qu'elle est vécue comme une nouvelle idylle, la teneur voluptueuse de ces instants est subitement anéantie par un incendie estival qui détruit les alentours de la maison, paysages et écuries. L'aube rend ce feu plus terrorisant encore et la lumière fade du petit matin se lève sur la vision insoutenable des ruines encore chaudes des villas et des cadavres des chevaux carbonisés. L'enchaînement de péripéties dans ce dernier opus redynamise, au final, la fougue amoureuse du Je qui, dans un monde chaotique et violent, aveuglant de lumières saturées, n'a pour dépasser ses tentations tétaniques que la tendresse langoureuse qu'il voue à Marie (Toussaint, 2009: 205). L'espace limité de l'île italienne concentre donc à son tour le puissant contraste qui meut l'œuvre entière en liant sur le mode pulsionnel et inéluctable Éros à la mort.

Habiles à appréhender les hantises de notre époque sans pour autant proposer de modèle de socialisation, les romans de Toussaint apportent une variété de nuances subtiles aux destinées sentimentales actuelles, grâce à un narrateur récurrent, aussi attachant qu'agaçant dans sa relation à l'Autre.

\section{RÉFÉRENCES BIBLIOGRAPHIQUES}

Barthes, R., (1980) La Chambre claire: note sur la photographie. Paris, GallimardSeuil.

Bessard-Banquy, O., (2003) Le roman ludique: Jean Echenoz, Jean-Philippe Toussaint, Éric Chevillard. Villeneuve d'Ascq, Presses Universitaires du Septentrion.

Demoulin, L. et P. Piret (dir.), (2010) Textyles. Dossier Jean-Philippe Toussaint. N ${ }^{\circ}$ 38.

Kaufmann, J.-C., (1999) Sociologie du couple. Paris, PUF.

Lipovetsky, G., (1997) La troisième femme: permanence et révolution du féminin. Paris, Gallimard.

Lipovetsky, G., (2006) Les Temps hypermodernes. Paris, Le Livre de Poche.

Pascal, B., (1941) L'Euvre de Pascal. Paris, Gallimard, Coll. Bibliothèque de la Pléiade.

Schoots, F., (1997) "Passer en douce à la douane". L'écriture minimaliste de Minuit: Deville, Echenoz, Redonnet et Toussaint. Amsterdam/Atlanta, Rodopi.

Toussaint, J.-P., (1985) La Salle de bain. Paris, Minuit.

Toussaint, J.-P., (1989) L'Appareil-photo. Paris, Minuit.

Toussaint, J.-P., (1997) La Télévision. Paris, Minuit.

Toussaint, J.-P., (2002) Faire l'amour. Paris, Minuit.

Toussaint, J.-P., (2005) Fuir. Paris, Minuit.

Toussaint, J.-P., (2009) La Vérité sur Marie. Paris, Minuit. 